\title{
Whole-exome sequencing identifies ADRA2A mutation in atypical familial partial lipodystrophy
}

\author{
Abhimanyu Garg, ${ }^{1}$ Shireesha Sankella, ${ }^{1}$ Chao Xing, ${ }^{2}$ and Anil K. Agarwal ${ }^{1}$ \\ 1Division of Nutrition and Metabolic Diseases, Department of Internal Medicine, and Center for Human Nutrition, \\ ${ }^{2}$ Department of Clinical Sciences and McDermott Center for Human Growth and Development, UT Southwestern Medical \\ Center, Dallas, Texas, USA.
}

\begin{abstract}
Despite identification of causal genes for various lipodystrophy syndromes, the molecular basis of some peculiar lipodystrophies remains obscure. In an African-American pedigree with a novel autosomal dominant, atypical familial partial lipodystrophy (FPLD), we performed linkage analysis for candidate regions and whole-exome sequencing to identify the disease-causing mutation. Affected adults reported marked loss of fat from the extremities, with excess fat in the face and neck at age 13-15 years, and developed metabolic complications later. A heterozygous g.112837956C $>$ T mutation on chromosome 10 (c.202C $>$ T, p.Leu68Phe) affecting a highly conserved residue in adrenoceptor $\alpha 2 A(A D R A 2 A)$ was found in all affected subjects but not in unaffected relatives. ADRA2A is the main presynaptic inhibitory feedback $G$ protein-coupled receptor regulating norepinephrine release. Activation of ADRA2A inhibits CAMP production and reduces lipolysis in adipocytes. As compared with overexpression of a wild-type ADRA2A construct in human embryonic kidney-293 cells and differentiated 3T3-L1 adipocytes, the mutant ADRA2A produced more cAMP and glycerol, which were resistant to the effects of the $\alpha 2$-adrenergic receptor agonist clonidine and the $\alpha 2$-adrenergic receptor antagonist yohimbine, suggesting loss of function We conclude that heterozygous p.Leu68Phe ADRA2A mutation causes a rare atypical FPLD, most likely by inducing excessive lipolysis in some adipose tissue depots.
\end{abstract}

\section{Introduction}

Lipodystrophies are rare disorders characterized by selective loss of body fat and predisposition to metabolic complications of insulin resistance, such as diabetes, hyperlipidemia, and hepatic steatosis (1). In the last 15 years, the molecular bases of many subtypes of genetic lipodystrophies have been unraveled (2-4). For example, mutations in 6 genes, LMNA, PPARG, PLIN1, AKT2, CIDEC, and LIPE, have been associated with familial partial lipodystrophy (FPLD) (5-12). More recently, whole-exome sequencing has been successful in elucidating causal genes for extremely rare subtypes of lipodystrophies, especially those due to de novo heterozygous variants (13-18). Despite this progress, the molecular genetic basis of some lipodystrophies in patients with peculiar phenotypes still remains obscure. Therefore, in this study, we investigated the molecular basis of an atypical FPLD in an African-American pedigree using SNP genotyping and whole-exome sequencing.

\section{Results}

Conflict of interest: The authors have declared that no conflict of interest exists.

Submitted: February 3, 2016 Accepted: May 17, 2016 Published: June 16, 2016

Reference information: JCI Insight. 2016;1(9):e86870. doi:10.1172/ji.insight.86870.

\section{Patients}

Clinical features of the affected adults with lipodystrophy are described below and summarized in Table 1 .

FPLD 122.3. This 46-year-old African-American male reported increased muscularity of the arms and legs at 13 years of age and excessive accumulation of fat in the dorsocervical region, requiring lipectomy at 33 and 40 years of age (Figure 1, A and B). He had hypertension, diabetes, dyslipidemia, gout, and hearing loss. He had obstructive sleep apnea, and at 41 years of age, underwent resection of the tongue and tonsillectomy. He complained of muscle cramps in the neck, stomach, and quadriceps region; heat intolerance; and excessive thirst. He smoked $0.5-1$ pack of cigarettes per day for 27 years. He had right hip joint 
Table 1. Clinical and laboratory characteristics of affected adults with atypical familial partial lipodystrophy

\begin{tabular}{|c|c|c|c|c|}
\hline Variable & FPLD 122.2 & FPLD 122.3 & FPLD 122.8 & Normal values \\
\hline Gender & Female & Male & Male & \\
\hline Age (yr) & 62 & 46 & 39 & \\
\hline Weight (kg) & 87.3 & 107.8 & 64.8 & \\
\hline BMI $\left(\mathrm{kg} / \mathrm{m}^{2}\right)$ & 33.3 & 36 & 24.3 & \\
\hline Diabetes (age of onset in yr) & $+(49)$ & $+(41)$ & $+(33)$ & \\
\hline Acanthosis nigricans & NA & + & - & \\
\hline Fasting glucose (mg/dl) & 191 & $155^{A}$ & 260 & $65-99$ \\
\hline Cholesterol (mg/dl) & 118 & $140^{\mathrm{A}}$ & 192 & $125-200$ \\
\hline Triglycerides (mg/dl) & 140 & $224^{\mathrm{A}}$ & 174 & $<150$ \\
\hline HDL cholesterol (mg/dl) & 46 & $37^{A}$ & 43 & $\geq 40$ \\
\hline Hemoglobin A1c (\%) & 9.4 & 7.5 & 13.6 & $<5.7$ \\
\hline Free fatty acids ( $\mathrm{mmol} / \mathrm{l})$ & NA & 0.55 & NA & $0.07-0.88$ \\
\hline Serum leptin (ng/ml) & 24.9 & 1.0 & 1.1 & \\
\hline Total body fat ${ }^{\complement}(\%)$ & NA & 11.6 & 9.1 & $12.0-38.0$ \\
\hline Arm fat ${ }^{\complement}(\%)$ & NA & 7.65 & 8.1 & NA \\
\hline Leg fat ${ }^{\complement}(\%)$ & NA & 8.1 & 5.65 & NA \\
\hline Trunk fat ${ }^{\mathrm{C}}(\%)$ & NA & 14.0 & 9.4 & NA \\
\hline Head fat ${ }^{\complement}(\%)$ & NA & 18.6 & 19.4 & NA \\
\hline
\end{tabular}

${ }^{A}$ Mean of 6 determinations over a period of 6 years. ${ }^{B} 5$ th to 95 th percentile values for African-American women, age $\geq 20$ years: $4.3-46.2 \mathrm{ng} / \mathrm{ml}$; for men, age $\geq 20$ years: $1.2-15.8 \mathrm{ng} / \mathrm{ml}$. 'Determined by dual-energy X-ray absorptiometry. Normal values for total body fat from Kelly et al. (19). To convert glucose values to SI units divide by 18. AST, aspartate aminotransferase; ALT, alanine aminotransferase; NA, not available.

replacement at 45 years of age. His mother and one younger brother also had lipodystrophy. He was taking nateglinide, metformin, sitagliptin, rosuvastatin, hydrochlorothiazide, carvedilol, losartan, esomeprazole, and promethazine. Examination revealed marked loss of s.c. fat from the upper and lower extremities, resulting in muscular appearance. Increased s.c. fat was present in the dorsal cervical area with a horizontal scar from previous surgery. He had deep-set eyes and a large tongue. He had reduced s.c. fat in the soles of his feet, with callouses laterally. His pulse rate ranged from 92 to 107 beats per minute (when he was not on carvedilol), and his blood pressure was 138/90 $\mathrm{mm} \mathrm{Hg}$. He had hepatomegaly $9.5 \mathrm{~cm}$ below the costal margin but no splenomegaly. Acanthosis nigricans was present in the groin. His fractionated plasma epinephrine was $73 \mathrm{pg} / \mathrm{ml}$ (normal value, $<50 \mathrm{pg} / \mathrm{ml}$ ) and norepinephrine was $769 \mathrm{pg} / \mathrm{ml}$ (normal value, $112-658 \mathrm{pg} / \mathrm{ml}$ ). Twenty-four hour urinary epinephrine was $13 \mu \mathrm{g}$ (normal value 2-24 $\mu \mathrm{g}$ ) and norepinephrine was $137 \mu \mathrm{g}$ (normal value 15-100 $\mu \mathrm{g}$ ). An electrocardiogram was normal.

FPLD 122.2. This 62-year-old African-American female reported masculine body since childhood and development of a buffalo hump at 13-14 years of age. She had menarche at 12.5 years of age and reported increased facial and body hair. She had removal of s.c. fat from the back of neck at 32 years of age. She also had hypertension, diabetes, and hyperlipidemia. She had a cerebrovascular accident at 40 years of age and has had claudication since 50 years of age. She had a partial hysterectomy at 23 years of age and cholecystectomy at 49 years of age. She smoked $0.5-1$ pack of cigarettes per day for 43 years. She was evaluated at 56 years of age when she was taking pioglitazone, metformin, hydrochlorothiazide, nasal fluticasone, and simvastatin.

FPLD 122.8. This 39-year-old African-American male reported being muscular all his life but noted increase accumulation of fat in the dorsocervical area at 13 years of age. He had ravenous appetite and was having episodic hot flashes lasting for 45 minutes. He also had diabetes, hypertension, and dyslipidemia. He smoked $0.5-1$ pack of cigarettes per day for 23 years. His pulse rate ranged from 74 to 88 beats per 

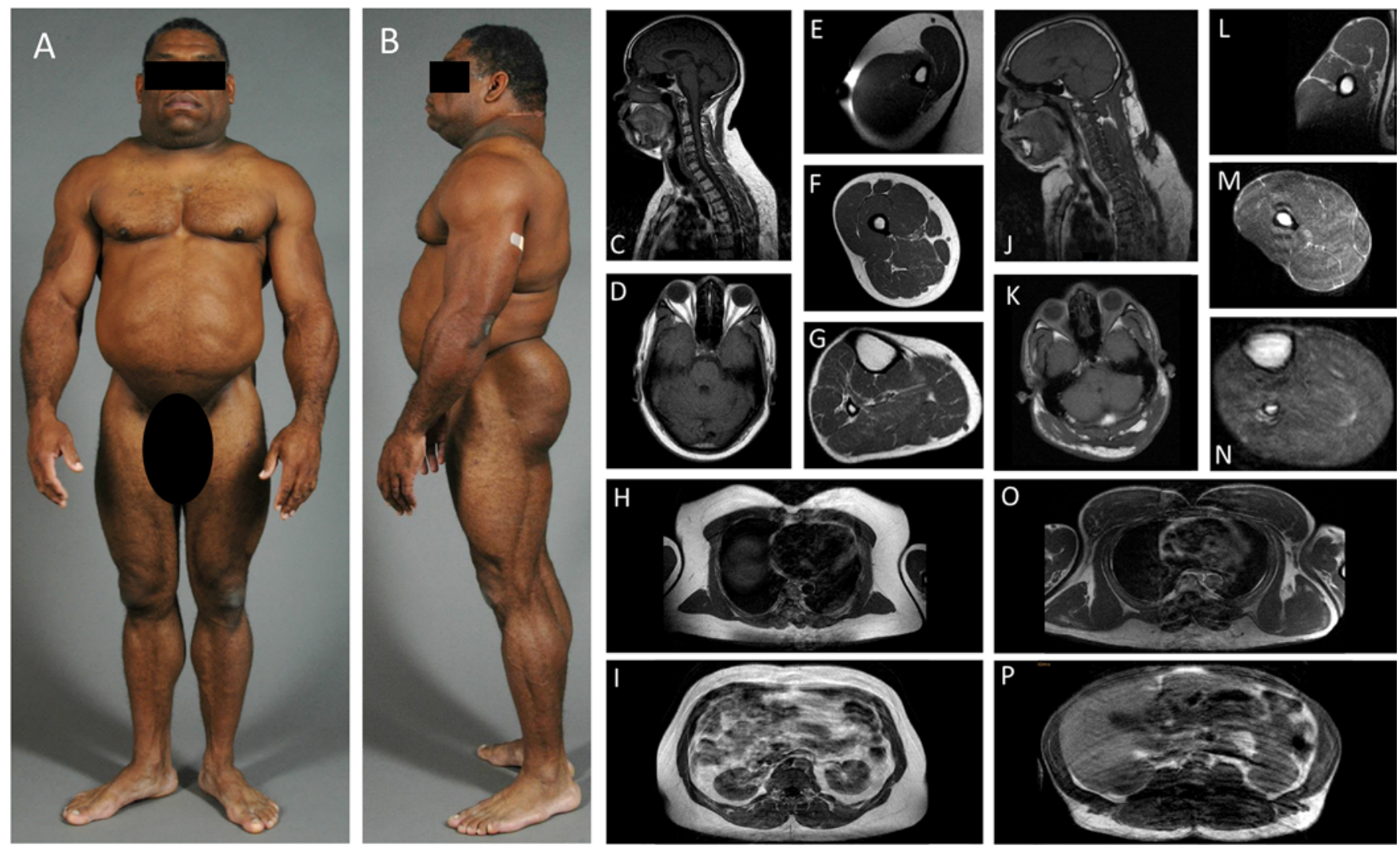

Figure 1. Clinical features of the proband (FPLD 122.3) and body fat distribution on whole-body MRI. (A) Anterior and (B) lateral view of the proband, showing marked loss of s.c. fat from the upper and lower extremities and the anterior region of the trunk and hips. Increased s.c. fat is present in the face and neck, both anteriorly and posteriorly. At the nape of the neck, a horizontal surgical scar from previous lipectomy is present. (C-I) T1-weighted sagittal and transaxial MRI images of a 47-year-old healthy African-American male with a BMI of $31.4 \mathrm{~kg} / \mathrm{m}^{2}$, and (J-P) corresponding images from the proband. Fat results in high signal intensity on T1-weighted images. (C) Sagittal MRI of the head and neck through midline shows a normal amount of s.c. fat in the scalp and slightly increased s.c. fat in the anterior and posterior neck region consistent with the BMI. (D) Axial MRI of the head through the nose shows normal s.c. fat in the posterior occipital and temporal regions and in the orbits. (E-G) Axial MRI images of the arm, thigh, and calf, showing a normal amount of s.c. fat. (H) Axial MRI image through the thorax, showing a normal amount of s.c. fat and a normal size of anterior and posterior muscles. (I) Axial MRI image through the abdomen at the level of the kidneys, showing normal fat in the s.c., intraperitoneal, and retroperitoneal (perinephric) region. (J) Sagittal MRI image of the head and neck of the proband through midline, showing some reduction of s.c. fat from the scalp but excess s.c. fat in the anterior and posterior neck regions. (K) Axial MRI image of the head through the nose, showing reduction of orbital fat and s.c. fat from the temporal region. (L) Axial MRI image of the arm, showing markedly reduced s.c. fat. (M) Axial MRI image of the thigh, showing near total lack of s.c. fat. The femoral bone has increased signal intensity suggesting normal bone marrow fat. (N) Axial MRI image of the calf, showing nearly absent s.c. fat. Tibial bone shows increased signal intensity. (0) Axial MRI image through the thorax, showing increased size of pectoralis and posterior thoracic muscles, including erector spinae but marked reduction of anterior s.c. fat. (P) Axial MRI image through the abdomen at the level of the kidneys, showing marked reduction in the anterior s.c. fat, with preservation of perirenal and intraperitoneal fat. Posterior s.c. fat remains well preserved.

minute, and he was normotensive. He also had marked loss of s.c. fat from the extremities, with increased fat deposition at the nape of the neck, submandibular, and submental regions. He had no acanthosis nigricans or hepatosplenomegaly.

The affected patients had a body fat distribution that was consistent with FPLD, i.e., marked loss of s.c. fat from the extremities and trunk but excessive accumulation of fat in the neck, posterior cervicothoracic region, and intra-abdominal region. Whole-body MRI of the proband (FPLD 122.3) and his affected brother (FPLD 122.8) further revealed unique loss of s.c. fat from the scalp and orbits and anterior truncal region but preservation of posterior s.c., perirenal, and intraperitoneal fat (Figure 1, C-P). The size of pectoralis and posterior thoracic muscles, including erector spinae, was increased. The affected patients also had reduced total body fat (19) and markedly reduced thickness of peripheral skin folds (biceps, triceps, forearm, thigh, and calf), but the skin fold thickness in the truncal region and chin was either normal or increased (refs. 20, 21, and Supplemental Figure 1; supplemental material available online with this arti- 


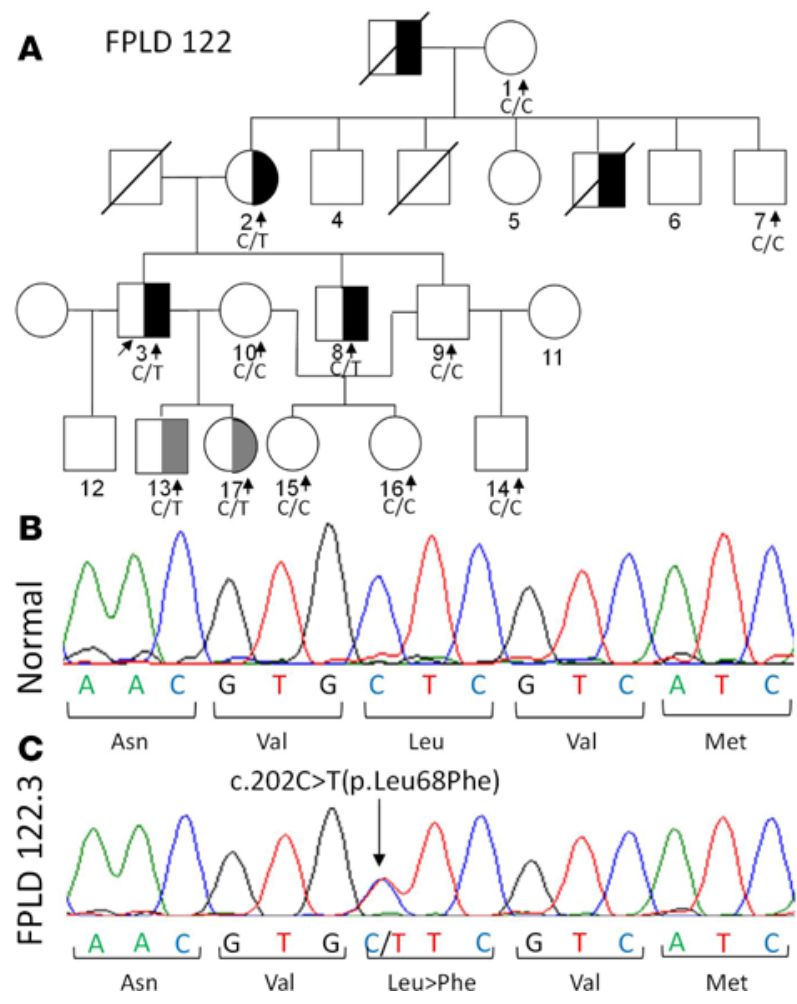

Figure 2. FPLD 122 pedigree and the disease-causing mutation. (A) FPLD 122 pedigree. Circles denote women, and squares denote men. Subjects with heterozygous c.202C $>T$ ADRA2A mutation are indicated with " $C / T$," and those with the wild-type alleles are indicated with " $C / C$." Symbols half-filled with black represent adult subjects with confirmed clinical diagnosis of lipodystrophy, whereas symbols half-filled with gray represent children in whom the clinical diagnosis of lipodystrophy could not be confirmed; white symbols indicate unaffected subjects. Vertical arrows indicate subjects for whom DNA was available, and a slanted arrow indicates the proband. Diagonal lines across the symbols indicate deceased subjects. (B) Sequence electropherogram from Sanger sequencing, showing normal sequence of ADRA2A. (C) Sequence electropherogram from FPLD 122.3, showing heterozygous c.202C $>T$ ( $p$.Leu68Phe) mutation.

cle; doi:10.1172/jci.insight.86870DS1). Sanger sequencing of the DNA of the proband, FPLD 122.3, for the known candidate genes for FPLD, LMNA, PPARG, PLIN1, and CIDEC, did not reveal any disease-causing variants.

Six adult subjects with unambiguous phenotype were used for linkage analysis - three were affected (individuals 2, 3, and 8), and three were unaffected (individuals 1, 7, and 9) (Figure 2A). Constrained by the pedigree size and the autosomal dominant mode of inheritance, the primary purpose of linkage analysis was not to identify linkage peaks of genome-wide significance, but to filter out genomic regions not shared by all the affected or shared between both the affected and the unaffected. Therefore, we used an arbitrary criterion of a lod score of 0.8 , which corresponds to half of the perfect lod score of $\log _{10}\left(1 / 0.5^{5}\right)=1.51$. Regions totaling approximately $300 \mathrm{Mb}$ had a linkage score greater than or equal to 0.8 . Using this filter, we could greatly reduce the number of candidate variants from the whole-exome sequencing data.

Whole-exome sequencing identified a total 50,452 variants in FPLD 122.2, FPLD 122.3, and FPLD 122.9, of which 11,724 were potentially functional variants (Supplemental Table 1). Filtering by minor allele frequency (MAF), linkage region, and genomic evolutionary rate profiling (GERP) score, we were left with 3 variants (Supplemental Table 2). Due to lack of segregation of the SLC25A1 and PVRL1 variants by Sanger sequencing, we concluded that a heterozygous g.112837956C $>\mathrm{T}$ variant (based on hg19) on chromosome 10 , leading to a c.202C $>$ T nucleotide change and corresponding p.Leu68Phe protein change in adrenoceptor $\alpha 2 \mathrm{~A}(A D R A 2 A)$, is the disease-causing variant in this family. Sanger sequencing further confirmed the segregation of the mutation in the family (Figure 2). In addition, an 8-year-old son and a 3-year-old daughter of the proband also harbored the ADRA2A mutant but did not show the lipodystrophy phenotype. Sanger sequencing of additional 64 unrelated patients with unexplained FPLD did not reveal any disease-causing mutation in $A D R A 2 A$.

Bioinformatics revealed that leucine at position 68 of ADRA2A is highly conserved across species (Figure $3 \mathrm{~A}$ ) and is present in the first transmembrane domain of this 7 -transmembrane $\mathrm{G}$ protein-coupled receptor according to one prediction (Figure $3 \mathrm{~B}$ ). In the human intra-abdominal and s.c. abdominal adipose tissue, the mRNA expression of $A D R A 2 A$ was approximately 9-fold and 17-fold higher, respectively, than that of $A D R A 2 C$, while $A D R A 2 B$ was undetectable (Figure $4, \mathrm{~A}$ and $\mathrm{B}$ ), and the expression of $A D R A 2 A$ was approximately 2 -fold higher in the s.c. abdominal fat compared with intra-abdominal fat (Figure 4C).

Next we tested the effects of overexpression of wild-type and mutant ADRA2A in cell model systems. Due to lack of a suitable antibody to ADRA2A, we generated expression constructs consisting of V5-epitoped tagged wild-type and mutant ADRA2A (to detect protein expression in cells) and expressed those in human embryonic kidney-293 (HEK-293) cells. The wild-type and mutant ADRA2A proteins were detectable on immunoblot (qualitatively) (Figure 4D) and showed similar posttranslational modification (glycosylation) for wild-type and mutant proteins (Figure 4E). Furthermore, the wild-type and mutant ADRA2A protein showed a similar subcellular (plasma membrane) localization pattern on immunofluorescence microscopy (Figure 4, F-H), suggesting that the p.Leu68Phe alteration does not destabilize the protein and that subcellular localization is similar to wild-type ADRA2A protein. 
A
H. sapiens
P.troglodytes
M.mulatta
B. taurus
M.musculus
R. norvegicus
G.gallus
D.rerio
D.melanogaster
C.elegans
H.sapiens (mutant)

TM1

LQVTLTLVCLAGLLMLLTVFGNV LVIIAVFTSRALK LQVTLTLVCLAGLLMLLTVFGNV LVI IAVFTSRALK LQVTLTLVCLAGLLMLLTVFGNV LVI IAVFTSRALK LQVTLTLVCLAGLLMLFTVFGNV LVI IAVFTSRALK LQVTLTLVCLAGLLMLFTVFGNV LVI IAVFTSRALK LQVTLTLVCLAGLLMLFTVFGNV LVI IAVFTSRALK LHTTVTLISLVGLLMLFTVFGNV LVI IAVFTSRALK LLVALPLSVAVGLLILLI IFGNV LVI IAVFTSRALR LTH IVIAS I IVT I LMI I IVVGNM LVI IA IATEKSLK LGEAVLTISSMLTVMLLI IFGNL LVVVTVYRDRKLR 45
80

80

80

65

80

80

72

54

147

53

80

B

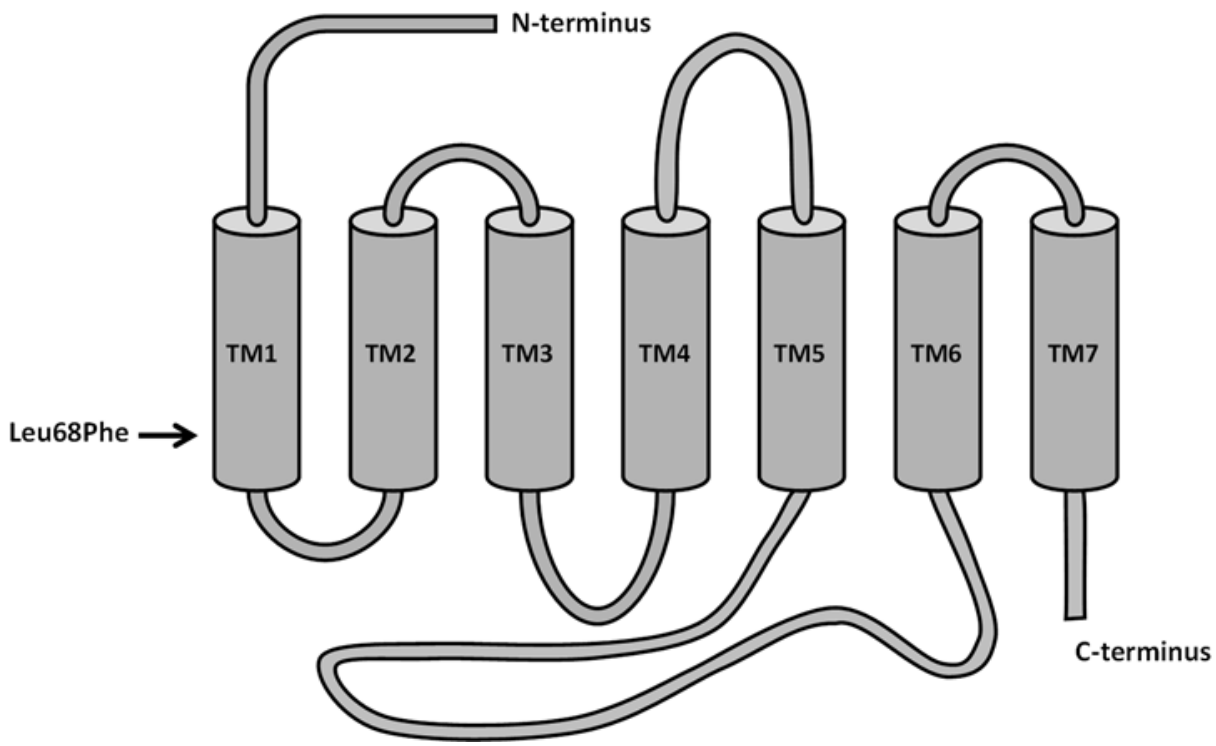

Figure 3. Conservation of the mutated leucine across species and the predicted model of human ADRA2A protein and the location of the mutation. (A) Alignment of partial ADRA2A sequences from the human (NP_000672.3), chimpanzee (XP_003312813.1), rhesus monkey (XP_001087738.2), cow (NP_776924.1), mouse (NP_031443.4), rat (NP_036871.3), chicken (XP_004942333.1), zebrafish (NP_997520.2), fruit fly (NP_001262714.1), and round worm (NP_001024569.1). The mutated residue leucine at position 68 (shown in red in bold font) is conserved among all the species. (B) The schematic of the ADRA2A protein (drawn as predicted by TMHMM2.0) shows 7 transmembrane domains and the location of the mutated leucine in the first transmembrane domain.

In our functional assays, the mutant ADRA2A resulted in slightly higher basal cAMP production in the transfected HEK-293 cells compared with the wild-type ADRA2A (mean [SD]: 14.2 [1.6] and 11.4 [2.8] $\mathrm{nM} / \mu \mathrm{g}$ protein, respectively), but this was not statistical significant $(P=0.069)$ (Figure $5, \mathrm{~A}$ and B). Further, cAMP synthesis in the cells transfected with mutant ADRA2A was resistant to inhibition by clonidine (omnibus $P=0.03$ ), with an $\mathrm{EC}_{50}$ of $48 \mu \mathrm{M}$, compared with cells transfected with wild-type protein $\left(\mathrm{EC}_{50}\right.$ of $\left.27 \mu \mathrm{M}\right)$ (Figure $5 \mathrm{~A}$ ). Similarly, cAMP synthesis in cells expressing the mutant ADRA2A was not sensitive to yohimbine (omnibus $P=0.046$ ), with an $\mathrm{EC}_{50}$ of $143 \mu \mathrm{M}$, compared with wild-type $\left(\mathrm{EC}_{50}\right.$ of $\left.79 \mu \mathrm{M}\right)$ (Figure $5 \mathrm{~B}$ ).

Transfection with mutant ADRA2A resulted in a higher rate of basal lipolysis, as evidenced by glycerol release in the medium from differentiated 3T3-L1 cells, compared with wild-type ADRA2A ( $P=$ 0.005 ) (Figure 5, C and D). Further, the glycerol release from the cells expressing mutant ADRA2A was resistant to suppression by clonidine (omnibus $P=0.021$ ), with an $\mathrm{EC}_{50}$ of $45 \mu \mathrm{M}$, compared with wildtype ADRA2A (EC ${ }_{50}$ of $3.5 \mu \mathrm{M}$ ) (Figure $5 \mathrm{C}$ ). Similarly, glycerol release from the cells expressing mutant ADRA2A was not sensitive to yohimbine (omnibus $P=0.024$ ), with an $\mathrm{EC}_{50}$ of $60 \mu \mathrm{M}$, compared with 35 $\mu \mathrm{M}$ in cell expressing wild-type protein (Figure $5 \mathrm{D}$ ). 
A

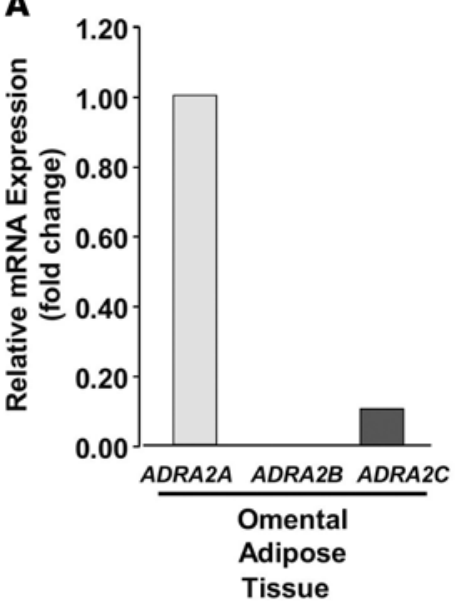

Tissue

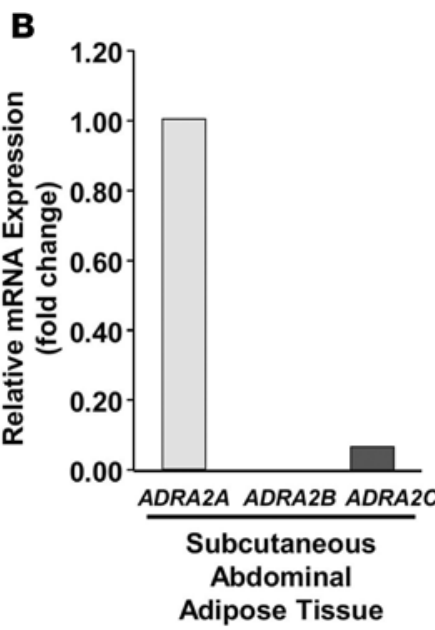

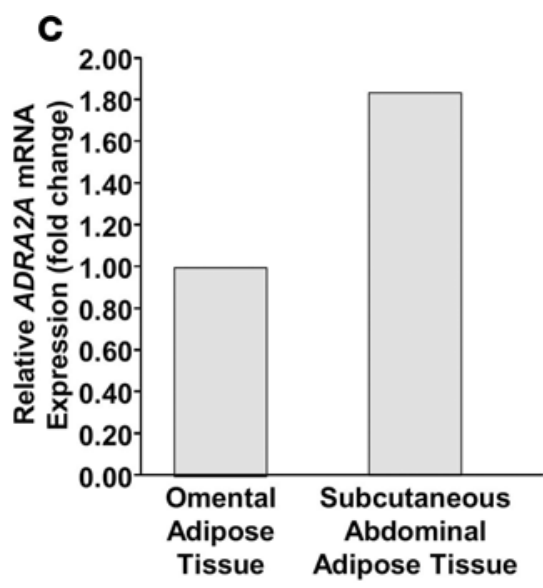

PNGase F

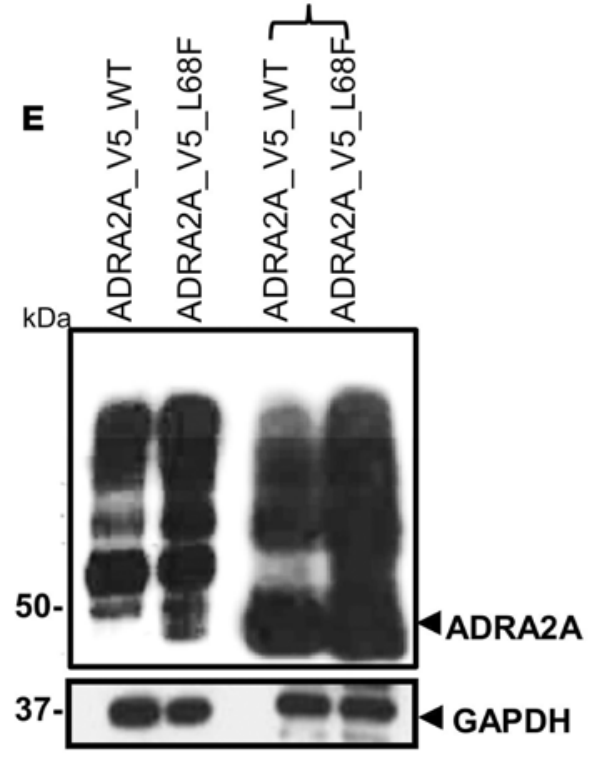

$\mathbf{F}$

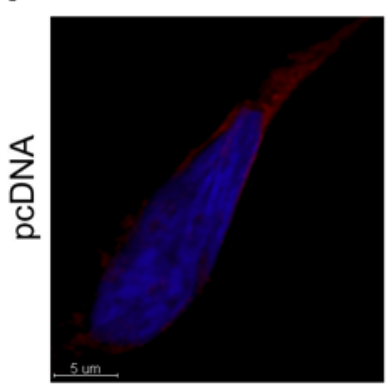

G

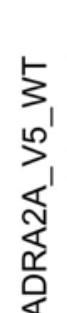

5 um

H
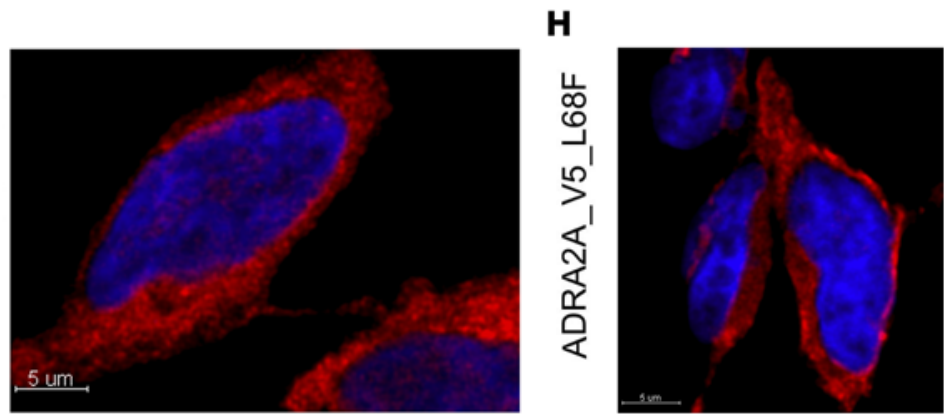
Figure 4. Relative mRNA expression of various ADRA2 isoforms in human adipose tissue, and expression and subcellular localization of ADRA2A wild-type and mutant proteins. (A-C) Relative mRNA expression of $A D R A 2 A, A D R A 2 B$, and $A D R A 2 C$ isoforms in the human omental and s.C. abdominal adipose tissue. (A) The expression of $A D R A 2 B$ and $A D R A 2 C$ isoforms was compared with that of $A D R A 2 A$ mRNA in omental adipose tissue, which was arbitrarily assigned a value of 1 . The $A D R A 2 A$ expression was 9.45 -fold higher than that of $A D R A 2 C$, while $A D R A 2 B$ was undetectable $\left(C_{t}>30\right)$. (B) The expression of $A D R A 2 B$ and $A D R A 2 C$ isoforms was compared with that of ADRA2A mRNA in s.c. abdominal adipose tissue, which was arbitrarily assigned a value of 1 . The $A D R A 2 A$ expression was 16.67 -fold higher than that of $A D R A 2 C$, while $A D R A 2 B$ was undetectable $\left(C_{t}>30\right)$. (C) The expression of ADRA2A in the s.c. abdominal adipose tissue was about 1.84-fold higher than in the omental adipose tissue. (D and E) Immunoblots for the expression of ADRA2A_V5_WT and ADRA2A_V5_L68F proteins as probed with V5 antibody. The immunoblots were probed (D) in the absence of peptide-N-glycosidase $\mathrm{F}$ (PNGase F) treatment (representative blot, $n=2$ ) and $(\mathbf{E}$ ) in the presence of PNGase $\mathrm{F}$ treatment (representative blot, $n=4$ ). PNGase $\mathrm{F}$ is an endoglycosidase that specifically removes $\mathrm{N}$-linked glycans from glycoproteins. GAPDH was included for protein loading control. The predicted molecular weight of human ADRA2A is approximately $50 \mathrm{kDa}$, but because it is a heavily glycosylated protein, it migrates slower than expected, as can be seen in $\mathbf{D}$. (E) Upon PNGase $F$ treatment, the deglycosylated protein reveals the approximate molecular weight of $50 \mathrm{kDa}$ for both for the wild-type and mutant ADRA2A. (F-H) Subcellular localization of human ADRA2A_V5_WT and ADRA2A_V5_L68F proteins expressed in HEK-293 cells. Proteins were fixed in $4 \%$ paraformaldehyde and incubated with primary antibody to V5 epitope followed by incubation with AlexaFluor 568-coupled fluorescent secondary antibody (red fluorescence) and counterstaining with DAPI (a nuclear stain; blue fluorescence). Cells were imaged for red and blue fluorescence using scanning confocal microscopy. Shown are single Z-slice images (Z-stacks were deconvolved using IMARIS software) for (F) pcDNA (vector control), (G) ADRA2A_V5_WT, and (H) ADRA2A_V5_L68F proteins. Both the ADRA2A_V5_WT and ADRA2A_V5_L68F localize to plasma membrane as expected (red fluorescence), confirming that there was no subcellular mislocalization of the mutant ADRA2A_V5_L68F protein. Numerous cells were examined visually, but only a few cells were processed.

\section{Discussion}

Adrenergic receptors belong to the superfamily of $\mathrm{G}$ protein-coupled receptors that mediate the biological actions of the endogenous catecholamines, epinephrine, and norepinephrine $(22,23)$. There are three main subtypes of these receptors, including $\alpha_{1}\left(\alpha_{1 \mathrm{~A}}, \alpha_{1 \mathrm{~B}}\right.$, and $\left.\alpha_{1 \mathrm{D}}\right), \alpha_{2}\left(\alpha_{2 \mathrm{~A}}, \alpha_{2 \mathrm{~B}}\right.$, and $\left.\alpha_{2 \mathrm{C}}\right)$, and $\beta\left(\beta_{1}, \beta_{2}\right.$, and $\left.\beta_{3}\right)(22$, 23). All have an extracellular amino terminus, 7 transmembrane domains, and an intracellular carboxy terminus $(24,25)$. ADRA2A receptors are constitutively active; however, upon binding to agonists, they activate heterotrimeric GTP-binding proteins (G proteins), which elicit suppression of second messenger signals, including cAMP. ADRA2A is highly expressed in the sympathetic nervous system and is the main presynaptic inhibitory feedback receptor controlling norepinephrine release (26). In adipocytes, ADRA2A activation inhibits cAMP production and reduces lipolysis (27).

Our study highlights an important effect of loss-of-function ADRA2A mutation causing atypical FPLD. Our functional studies reveal that the mutant ADRA2A protein fails to suppress the production of cAMP in HEK-293 cells and increases lipolysis in differentiated 3T3-L1 adipocytes, suggesting that substitution of leucine at position 68 to phenylalanine results in loss of function of ADRA2A. Further, cAMP production and lipolysis from ADRA2A mutant-expressing cells were resistant to clonidine and yohimbine. These data suggest that excessive lipolysis from adipose tissue resulting from ADRA2A mutation may be the main mechanism causing lipodystrophy.

Higher expression of $A D R A 2 A$ compared with $A D R A 2 B$ and $A D R A 2 C$ in human adipose tissue suggests an important role of this isoform in adipocyte biology. Further, nearly 2-fold higher expression of $A D R A 2 A$ in the human s.c. abdominal fat compared with omental fat is consistent with the loss of adipose tissue from the anterior truncal region in our patients with ADRA2A mutation while omental adipose tissue is spared. However, it is unclear why ADRA2A mutation results in atrophy of some adipose tissue depots located in the periphery, while the others located in the neck, face, and intra-abdominal region are spared and in fact undergo hypertrophy. Further studies are required to assess specific functions of $A D R A 2 A$ in various human adipose tissue depots.

The likelihood of digenic etiology is low in our patient - given the evident cosegregation of the $A D R A 2 A$ variant and the phenotype across 3 generations, the odds of a second hit not linked to $A D R A 2 A$ but cosegregated with the $A D R A 2 A$ mutation in the 4 affected subjects of the affected founder are low $\left[0.5^{4} /\left(1-0.5^{4}\right)=0.067\right]$. Lack of ADRA2A mutations in other unexplained patients with FPLD suggests that this is a rare subtype of FPLD. This is similar to the previously reported mutations in $A K T 2$ and CIDEC in single families with FPLD $(9,10)$.

Genetic deletion of Adra2a in mice results in increased heart rate (28-31), normal or elevated blood pressure $(28,30,32)$, and an increase in plasma norepinephrine $(30,33)$ but no impairment of cardiac function (33). Although, previous studies in $\mathrm{Adra2a^{-1 }}$ mice did not thoroughly analyze adipose tissue distribution, since Adra2a expression is low in mouse adipose tissue $(26,34)$, lipodystrophy may not be expected. All adult affected subjects from the FPLD 122 family had hypertension, but an increase in heart rate was not a consistent finding. The proband did have slightly high plasma and urinary norepinephrine levels. 
A

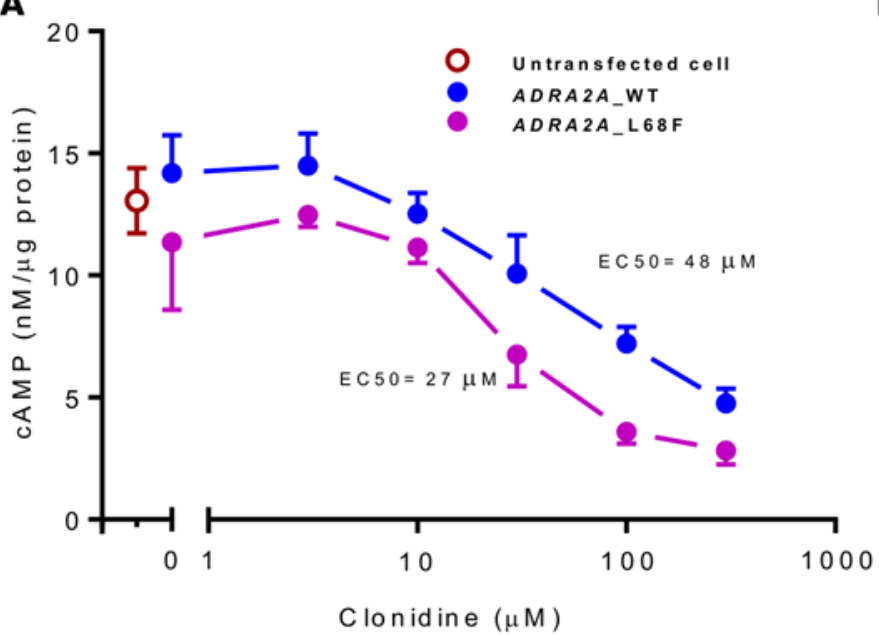

B

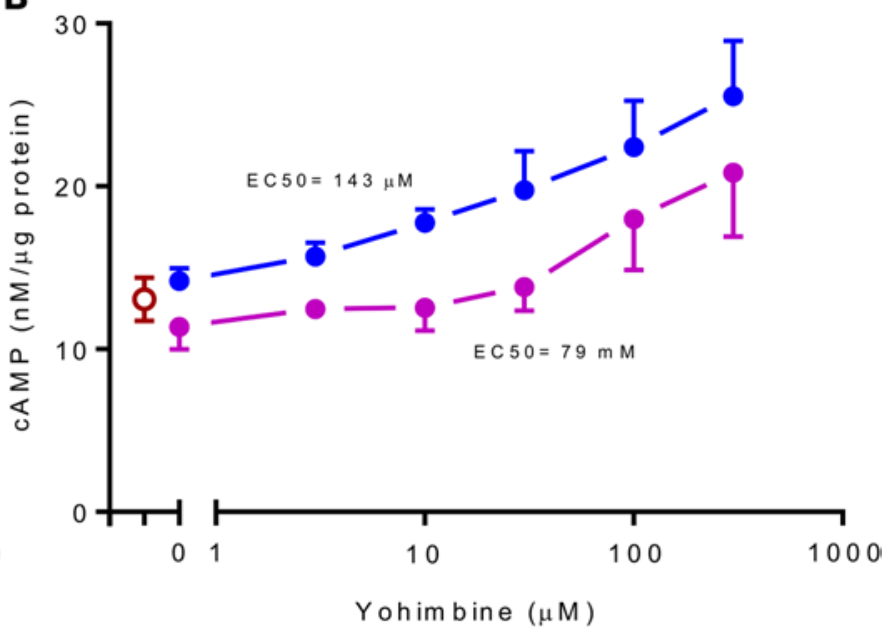

C

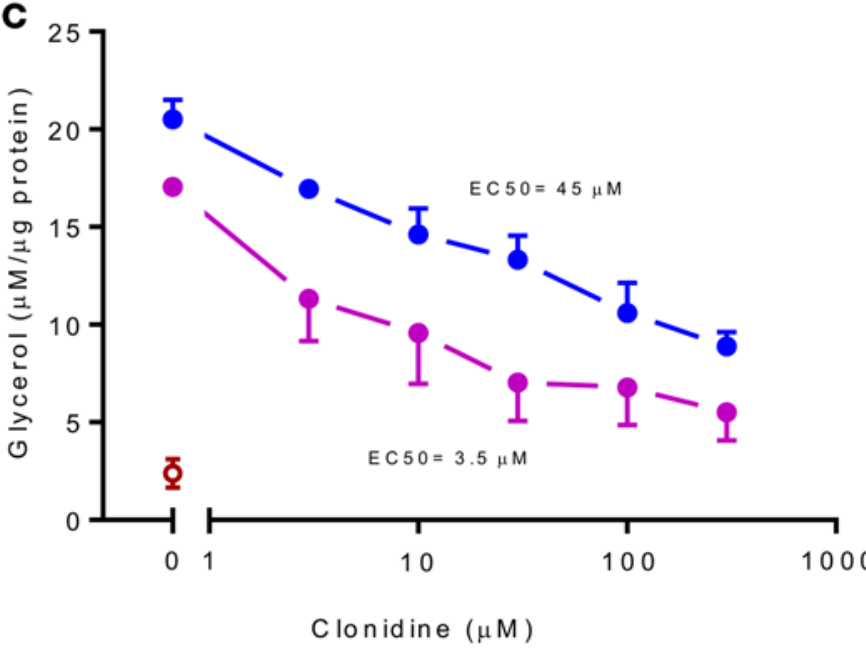

D

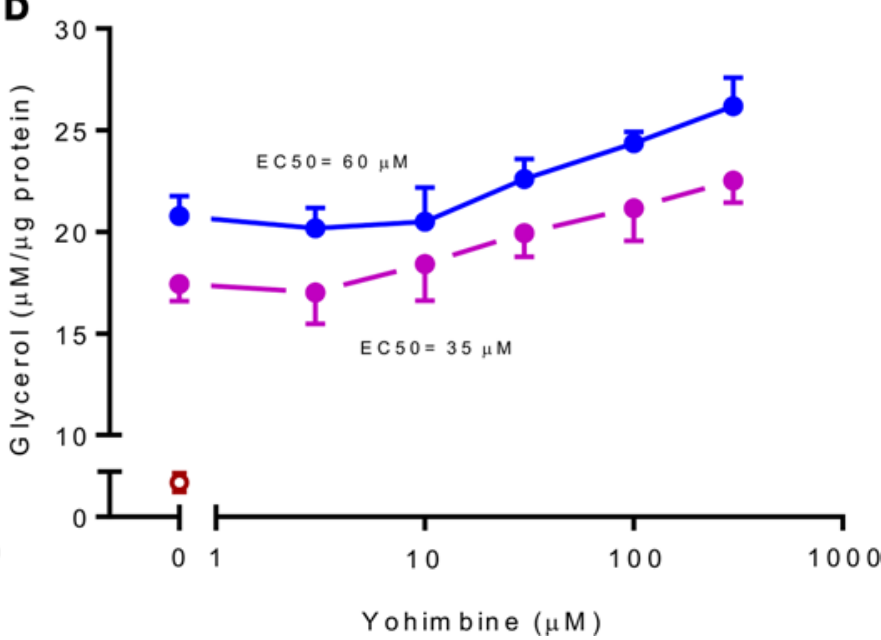

Figure 5. Effects of overexpression of wild-type and mutant ADRA2A in HEK-293 and 3T3-L1 preadipocyte cells on cAMP and glycerol production, respectively - response to clonidine and yohimbine. (A and B) HEK-293 cells were transfected with ADRA2A_WT and ADRA2A_L68F containing V5 epitope tag at the amino terminus. The production of cAMP was measured in the presence of various concentrations of clonidine or yohimbine by cAMPGlo assay and normalized to protein. Dose response curves for $(\mathbf{A})$ clonidine or (B) yohimbine were plotted as a semi-log curves and expressed as nM cAMP produced per $\mu$ g protein (mean $\pm \mathrm{SEM}, n=4$ ). For clonidine, $A D R A 2 A \_\mathrm{WT}$ has an $\mathrm{EC}_{50}$ value of $27 \mu \mathrm{M}$ and $A D R A 2 A_{-} L 68 \mathrm{~F}$ has an EC $\mathrm{E}_{50}$ value of $48 \mu \mathrm{M}$. For yohimbine, $A D R A 2 A \_W T$ has an $\mathrm{EC}_{50}$ value of $79 \mu \mathrm{M}$ and $A D R A 2 A \_L 68 \mathrm{~F}$ has an $\mathrm{EC}_{50}$ value of $143 \mu \mathrm{M}$. (C and D) ADRA2A_WT and ADRA2A_L68F containing V5 epitope tag at the amino terminus were expressed in 3T3-L1 cells. Twenty-four hours after transfection, the cells were allowed to differentiate into adipocytes using standard differentiation protocol (see the Methods for details). The cells were differentiated for 8 days and incubated with various concentrations of (C) clonidine or (D) yohimbine dissolved in dimethylsulfoxide for $\mathbf{2 4}$ hours. Released glycerol in the media was measured, normalized to protein, and expressed as $\mu \mathrm{M}$ glycerol released/ $\mu$ g protein (mean $\pm \mathrm{SEM}, n=3$ for clonidine and $n=4$ for yohimbine). The expression of cAMP or release of glycerol in the cells not treated with constructs or vehicle is also plotted in each panel. cAMP production or glycerol release from mutant ADRA2A compared with wild-type ADRA2A was significant using mixed-effect model, which assessed genotype, dose, and genotype $\times$ dose interaction as fixed effects, with each experiment modeled as a random effect. The $\mathrm{EC}_{50}$ values were determined by using GraphPad Prism version 6.04. Since our $\mathrm{X}$ coordinates are plotted as logarithms, and since the log of 0 is undefined, we approximated this point with an $X$ coordinate of $0.01 \mu M$, about 2 log units below the lowest "real" X value. For clonidine, ADRA2A_WT has an $\mathrm{EC}_{50}$ value of $3.5 \mu \mathrm{M}$ and $A D R A 2 A \_L 68 \mathrm{~F}$ has an $\mathrm{EC}_{50}$ value of $45 \mu \mathrm{M}$. For yohimbine, ADRA2A_WT has an $\mathrm{EC}_{50}$ value of $35 \mu \mathrm{M}$ and $A D R A 2 A_{-}$L68F has an $\mathrm{EC}_{50}$ value of $60 \mu \mathrm{M}$. Analyses were performed with SAS 9.4 (SAS Institute). An omnibus $P$ value of less than 0.05 was considered statistically significant.

Nearly all previously reported molecular defects resulting in lipodystrophy have been in pathways involved in storage of triglycerides in lipid droplets of adipocytes (AGPAT2, BSCL2, CAV1, PTRF, PLIN1, CIDEC, LIPE), adipocyte differentiation (AGPAT2, BSCL2, PPARG, AKT2), or adipocyte cell death ( $L M N A$, ZMPSTE24) $(2,4,11,12)$. Adding to this growing list, now, is the $A D R A 2 A$ mutation, which causes atypical FPLD mainly by altering lipolysis. In addition, PLIN1 mutations cause FPLD by increasing lipolysis by recruiting hormone-sensitive lipase to the surface of lipid droplets to initiate lipolysis $(8,35,36)$. We report a G protein-coupled receptor pathway involved in causing atypical FPLD in which excessive lipolysis 
results in loss of adipose tissue. Our data also suggest a potential targeted therapy for patients, such as the ADRA2A agonist, clonidine, which may suppress lipolysis and potentially limit or prevent fat loss.

\section{Methods}

Body fat distribution studies. Anthropometric measurements, whole-body MRI, and dual-energy x-ray absorptiometry (Hologic Delphi W densitometer; Hologic Inc.) were performed to determine total body fat and its distribution as described previously (37).

Sanger sequencing. Genomic DNA was isolated from peripheral blood using the Easy-DNA kit (Invitrogen). The coding regions and the splice site junctions of LMNA, PPARG, PLIN1, and CIDEC were amplified with gene-specific primers as described earlier (38). The PCR product was purified to remove primers and dNTPs and Sanger sequenced using ABI Prism 3100. The exon and surrounding 5' and 3 regions of $A D R A 2 A$ were amplified with gene-specific primers. In addition, 64 patients with unexplained FPLD were genotyped for $A D R A 2 A$ using Sanger sequencing. We also performed Sanger sequencing of the candidate variants in PVRL1 and SLC25A1 among the family members to investigate for segregation with the phenotype.

Linkage analysis. Six adult subjects (three affected and three unaffected) from the FPLD 122 pedigree (1, 2, 3, 7, 8, and 9) underwent genotyping on the Illumina HumanOmnil-Quad SNP array. We chose 7,296 SNPs spaced approximately 2 per centiMorgan from among the approximately 1 million to construct a sparse linkage map (39). We performed multipoint model-based linkage analysis under a dominant inheritance pattern, with a penetrance of 0.9 , sporadic rate of 0.001 , and disease-predisposing allele frequency of 0.001 using MERLIN (40).

Whole-exome sequencing. Two affected (FPLD 122.2 and FPLD 122.3) and one unaffected (FPLD 122.9) subject underwent whole-exome sequencing using the SureSelect Human All Exon V4 kit on the Illumina platform. Sequencing read length was paired-end $2 \times 100 \mathrm{bp}$. Sequences were aligned to the human reference genome b37, and variants were called using the Genome Analysis Toolkit HaplotypeCaller and annotated using snpEff. We hypothesized that an autosomal dominant mutation was most likely and therefore filtered for rare - absent in the 1000 Genomes and MAF $<0.1 \%$ in the Exome Aggregation Consortium database - missense, nonsense, splicing, or frameshift mutations located in linkage regions with lod scores greater than 0.8. We filtered for conservative mutations with GERP++ scores greater than 2.0 (41). We also used Sanger sequencing to confirm segregation of the candidate variants within the pedigree.

Expression of ADRA2 mRNA isoforms in human adipose tissue, and expression and subcellular localization of mutant ADRA2A protein. We studied mRNA expression of various ADRA2 isoforms in s.c. abdominal and omental human adipose tissue (Supplemental Methods and Supplemental Tables 3 and 4). We also introduced the mutation encoding p.Leu68Phe into human $A D R A 2 A \mathrm{cDNA}$, expressed these constructs in HEK-293 cells, and examined protein expression and subcellular localization by immunoblotting and immunofluorescence analyses (Supplemental Methods and Supplemental Table 3).

Deglycosylated and glycosylated immunoblot analysis of human ADRA2A protein. Twenty-four hours after transfection, HEK-293 cells (ATCC) were lysed in lysis buffer (10 mM Tris, 1 mM EDTA, pH 7.4, with protease inhibitor cocktail) and sonicated using a Vibra cell VCX 130 sonicator (Sonics) at 30\% amplitude, with 1 to 2 pulses for 15 seconds each. The supernatant was spun at 1,000 $g$ for 5 minutes to remove debris followed by centrifugation of the supernatant at $15,000 \mathrm{~g}$ for 30 minutes at $16^{\circ} \mathrm{C}$. Total protein was measured in the cleared supernatant by Bio-Rad DC protein assay (Bio-Rad). A total of $10 \mu \mathrm{g}$ of the protein was treated with peptide-N-glycosidase F (PNGase F; New England Biolabs) according to the manufacturer's protocol. PNGase F deglycosylates a native glycoprotein by cleaving between the innermost acetylglucosamine (GlcNAc) and asparagine residues of high mannose, hybrid, and complex oligosaccharides from $\mathrm{N}$-linked glycoproteins. The ADRA2A protein was denatured by adding $1 \mu 1$ of $10 \times$ glycoprotein denaturing buffer and boiling at $100^{\circ} \mathrm{C}$ for 10 minutes. After cooling the reaction mixture on ice, $2 \mu 1$ of $10 \%$ NP-40, $2 \mu$ of 10X G7 reaction buffer, and 500 units of PNGase F enzyme were added. The samples were incubated at $37^{\circ} \mathrm{C}$ for 1 hour and then separated on 10\% SDS-PAGE (Bio-Rad) along with untreated samples followed by transfer onto polyvinylidene fluoride membranes (Millipore). The membrane was blocked with $5 \%$ non-fat dry milk containing $0.2 \%$ Tween 20 for 1 hour at room temperature. The membrane was then incubated with anti-V5 primary antibody (catalog 46-0705; lot 1138580, Life Technologies) overnight at $4^{\circ} \mathrm{C}$ at 1:2,000 dilution and then washed with $1 \mathrm{X}$ TBS-T (Tris-buffered saline and Tween 20) 3 times and incubated with secondary antibody to IgG (goat anti-mouse-IgG conjugated to HRP) at a 1:5,000 dilution 
for 1 hour at room temperature (Santa Cruz Biotechnology) and detected with Immobilon Western Chemiluminescent HRP substrate (Millipore) and exposed onto X-ray films.

The same blot was stripped using Restore Western blot stripping buffer (Thermo Fisher Scientific) according to the manufacturer's protocol and reprobed with GAPDH antibody at 1:5,000 dilution (mouse monoclonal, Ambion).

Immunofluorescence microscopy. HEK-293 cells were plated on collagen-coated coverslips in a 6-well plate and transfected with pcDNA (vector) or $A D R A 2 A \_V 5 \_W T$ or $A D R A 2 A \_V 5 \_L 68$ F. Twenty-four hours after transfection cells were fixed in $4 \%$ paraformaldehyde. Cells were treated with blocking solution without NP-40. Cells were washed 3 times for 5 minutes each with PBS and then incubated with primary antibody to V5 epitope (Life Technologies, dilution of 1:1,000, Life Technologies) for 60 minutes at room temperature. Cells were then washed 3 times for 5 minutes each with PBS and incubated with AlexaFluor 568-coupled fluorescent secondary antibody (Life Technologies) for 60 minutes at room temperature. After incubation, cells were washed 3 times for 5 minutes each with PBS, costained with DAPI during the washes, and mounted on a glass slide with Aqua Poly/Mount (Polysciences Inc.). Cell images were acquired by a DeltaVision RT Deconvolution Microscope (Applied Precision, LLC). Z-stack images for red and blue fluorescence were acquired in approximately 0.15 -micron increments at $\times 60$ magnification and were deconvolved using SoftWoRx (Applied Precision). Immunofluorescent images were processed with a DeltaVision workstation (Applied Precision).

Functional assay for the mutant $A D R A 2 A$. The effects of the overexpression of the mutant and wild-type ADRA2A constructs (Supplemental Methods) on cAMP release were studied in HEK-293 cells (ATCC) and on lipolysis were studied in murine 3T3-L1 preadipocytes. The HEK-293 cells were plated at a density of $2 \times 10^{5}$ cells per well of a 96-well plate and allowed to attach overnight in low glucose Dulbecco's Modified Eagle's medium supplemented with $10 \%$ fetal bovine serum, $1 \%$ penicillin, and streptomycin. The next day, the cells were transfected with $500 \mathrm{ng}$ of pcDNA (vector), wild-type (ADRA2A_V5_WT), and mutant (ADRA2A_V5_L68F) plasmids in Optimem medium using Lipofectamine 2000 (Life Technologies). Six hours after transfection, the Optimem medium was replaced with Dulbecco's Modified Eagle's medium. Twenty-four hours after transfection, the cells were treated with $(0,3,10,30,100$, or $300 \mu \mathrm{M})$ clonidine (an ADRA2A agonist) or yohimbine (an ADRA2A antagonist) for 24 hours. The cells were lysed using lysis buffer, and intracellular cAMP levels were measured using the cAMP-Glo Max assay (Promega). The lysate was centrifuged for 10 minutes at $16,000 \mathrm{~g}$ at $4^{\circ} \mathrm{C}$, and the protein concentration of the supernatant was measured using DC protein assay (Bio-Rad).

The transfection of 3T3-L1 cells (ATCC) was performed at a density of $1 \times 10^{4}$ cells per well using 100 ng of pcDNA. Twenty-four hours after transfection, the cells were induced to differentiate into adipocytes by addition of $0.5 \mathrm{mM}$ 3-isobutyl 1-methylxanthine, $10 \mu \mathrm{g} / \mathrm{ml}$ insulin, $10 \mu \mathrm{M}$ dexamethasone, and $1 \mu \mathrm{M}$ rosiglitazone for 48 hours followed by addition of culture medium containing insulin every 48 hours for 5 additional days. After differentiation, cells were incubated with clonidine or yohimbine for 24 hours. The media were collected and glycerol was measured using a lipolysis assay kit (Zen-Bio Inc.).

The half maximal effective concentrations $\left(\mathrm{EC}_{50}\right)$ for glycerol and $\mathrm{cAMP}$ response to clonidine and yohimbine were determined using GraphPad prism 6 software version 6.04.

Statistics. For comparing data on cAMP and glycerol production from the mutant and wild-type ADRA2A-expressing cell lines in response to increasing doses of clonidine and yohimbine, the mixed effects model was employed using SAS version 9.4 (SAS Institute). The model assessed genotype, dose, and genotype $\times$ dose interaction as fixed effects with each experiment modeled as a random effect. Analyses were performed with SAS 9.4 (SAS Institute) and GraphPad Prism version 6 software, version 6.04. An omnibus $P$ value of less than 0.05 was considered statistically significant.

Study approval. The present studies in humans were reviewed and approved by the institutional review board of UT Southwestern Medical Center. All subjects provided written informed consent prior to participation in the study.

\section{Author contributions}

AG, CX, and AKA designed the study. AG collected clinical data on the pedigree. CX performed linkage analysis and analyzed whole-exome sequencing data. SS and AKA performed functional experiments. All authors wrote, commented on, and edited the manuscript. 


\section{Acknowledgments}

We thank Michael W. Schwartz, from the University of Washington, Seattle, for referring the proband to us; Claudia Quittner, for nursing support; Pei-Yun Tseng and Katie Tunison, for illustrations and mutational screening; the McDermott Center Sequencing and Bioinformatics Cores for sequencing and analysis; and Jonathan Rios, for his suggestions and review of the manuscript. This work was supported by NIH grant RO1-DK105448, Clinical and Translational Science Award grants UL1RR024982 and UL1TR001105, and the Southwest Medical Foundation.

Address correspondence to: Abhimanyu Garg, UT Southwestern Medical Center, 5323 Harry Hines Blvd., K5.214, Dallas, Texas 75390-8537, USA. Phone: 214.648.2895; E-mail: abhimanyu.garg@utsouthwestern.edu.

1. Garg A. Acquired and inherited lipodystrophies. N Engl J Med. 2004;350(12):1220-1234.

2. Garg A. Clinical review\#: Lipodystrophies: genetic and acquired body fat disorders. J Clin Endocrinol Metab. 2011;96(11):33133325.

3. Garg A. Genetic lipodystrophies. In: Rimoin DL, Connor JM, Pyeritz RE, Korf BR, eds. Emery and Rimoin's Principles and Practice of Medical Genetics. 6th ed. London, United Kingdom: Churchill Livingstone, Elsevier; 2013:1-16.

4. Robbins AL, Savage DB. The genetics of lipid storage and human lipodystrophies. Trends Mol Med. 2015;21(7):433-438.

5. Cao H, Hegele RA. Nuclear lamin A/C R482Q mutation in Canadian kindreds with Dunnigan-type familial partial lipodystrophy. Hum Mol Genet. 2000;9(1):109-112.

6. Shackleton S, et al. LMNA, encoding lamin A/C, is mutated in partial lipodystrophy. Nat Genet. 2000;24(2):153-156.

7. Agarwal AK, Garg A. A novel heterozygous mutation in peroxisome proliferator-activated receptor-g gene in a patient with familial partial lipodystrophy. J Clin Endocrinol Metab. 2002;87(1):408-411.

8. Gandotra S, et al. Perilipin deficiency and autosomal dominant partial lipodystrophy. N Engl J Med. 2011;364(8):740-748

9. George S, et al. A family with severe insulin resistance and diabetes due to a mutation in AKT2. Science. 2004;304(5675):13251328.

10. Rubio-Cabezas $\mathrm{O}$, et al. Partial lipodystrophy and insulin resistant diabetes in a patient with a homozygous nonsense mutation in CIDEC. EMBO Mol Med. 2009;1(5):280-287.

11. Albert JS, et al. Null mutation in hormone-sensitive lipase gene and risk of type 2 diabetes. N Engl J Med. 2014;370(24):23072315.

12. Farhan SM, et al. A novel LIPE nonsense mutation found using exome sequencing in siblings with late-onset familial partial lipodystrophy. Can J Cardiol. 2014;30(12):1649-1654.

13. Weedon MN, et al. An in-frame deletion at the polymerase active site of POLD1 causes a multisystem disorder with lipodystrophy. Nat Genet. 2013;45(8):947-950.

14. Dyment DA, et al. Mutations in PIK3R1 cause SHORT syndrome. Am J Hum Genet. 2013;93(1):158-166.

15. Thauvin-Robinet C, et al. PIK3R1 mutations cause syndromic insulin resistance with lipoatrophy. Am J Hum Genet. 2013;93(1):141-149.

16. Payne F, et al. Mutations disrupting the Kennedy phosphatidylcholine pathway in humans with congenital lipodystrophy and fatty liver disease. Proc Natl Acad Sci U S A. 2014;111(24):8901-8906

17. Garg A, Kircher M, Del Campo M, Amato RS, Agarwal AK, University of Washington Center for Mendelian Genomics Whole exome sequencing identifies de novo heterozygous CAV1 mutations associated with a novel neonatal onset lipodystrophy syndrome. Am J Med Genet A. 2015;167A(8):1796-1806.

18. Masotti A, et al. Keppen-Lubinsky syndrome is caused by mutations in the inwardly rectifying $\mathrm{K}^{+}$channel encoded by $\mathrm{KCNJ} 6$. Am J Hum Genet. 2015;96(2):295-300.

19. Kelly TL, Wilson KE, Heymsfield SB. Dual energy X-Ray absorptiometry body composition reference values from NHANES PLoS One. 2009;4(9):e7038.

20. Jackson AS, Pollock ML. Generalized equations for predicting body density of men. Br J Nutr. 1978;40(3):497-504.

21. Jackson AS, Pollock ML, Ward A. Generalized equations for predicting body density of women. Med Sci Sports Exerc. 1980;12(3):175-181.

22. Dorn GW. Adrenergic signaling polymorphisms and their impact on cardiovascular disease. Physiol Rev. 2010;90(3):1013-1062.

23. Hein L. Adrenoceptors and signal transduction in neurons. Cell Tissue Res. 2006;326(2):541-551.

24. Lafontan M, Berlan M. Fat cell adrenergic receptors and the control of white and brown fat cell function. J Lipid Res. 1993;34(7):1057-1091.

25. Rosengren AH, et al. Overexpression of $\alpha 2 \mathrm{~A}$-adrenergic receptors contributes to type 2 diabetes. Science. 2010;327(5962):217220.

26. Lafontan M, Berlan M. Fat cell alpha 2-adrenoceptors: the regulation of fat cell function and lipolysis. Endocr Rev. 1995;16(6):716-738.

27. Stich V, et al. Activation of antilipolytic alpha(2)-adrenergic receptors by epinephrine during exercise in human adipose tissue. Am J Physiol. 1999;277(4 pt 2):R1076-R1083.

28. Altman JD, et al. Abnormal regulation of the sympathetic nervous system in $\alpha 2 \mathrm{~A}$-adrenergic receptor knockout mice. Mol Pharmacol. 1999;56(1):154-161.

29. Hein L, Limbird LE, Eglen RM, Kobilka BK. Gene substitution/knockout to delineate the role of $\alpha 2$-adrenoceptor subtypes in mediating central effects of catecholamines and imidazolines. Ann N Y Acad Sci. 1999;881:265-271.

30. Makaritsis KP, et al. Sympathoinhibitory function of the $\alpha(2 \mathrm{~A})$-adrenergic receptor subtype. Hypertension. 1999;34(3):403-407. 
31. Brede M, et al. Feedback inhibition of catecholamine release by two different $\alpha 2$-adrenoceptor subtypes prevents progression of heart failure. Circulation. 2002;106(19):2491-2496.

32. Brum PC, Kosek J, Patterson A, Bernstein D, Kobilka B. Abnormal cardiac function associated with sympathetic nervous system hyperactivity in mice. Am J Physiol Heart Circ Physiol. 2002;283(5):H1838-H1845.

33. Hein L, Altman JD, Kobilka BK. Two functionally distinct $\alpha 2$-adrenergic receptors regulate sympathetic neurotransmission. Nature. 1999;402(6758):181-184.

34. Castan I, et al. Antilipolytic effects of $\alpha 2$-adrenergic agonists, neuropeptide Y, adenosine, and PGE1 in mammal adipocytes. Am J Physiol. 1994;266(4 pt 2):R1141-R1147.

35. Gandotra S, Lim K, Girousse A, Saudek V, O'Rahilly S, Savage DB. Human frame shift mutations affecting the carboxyl terminus of perilipin increase lipolysis by failing to sequester the adipose triglyceride lipase (ATGL) coactivator AB-hydrolase-containing 5 (ABHD5). J Biol Chem. 2011;286(40):34998-35006.

36. Kozusko K, et al. Clinical and molecular characterization of a novel PLIN1 frameshift mutation identified in patients with familial partial lipodystrophy. Diabetes. 2015;64(1):299-310.

37. Ji H, Weatherall P, Adams-Huet B, Garg A. Increased skeletal muscle volume in women with familial partial lipodystrophy, Dunnigan variety. J Clin Endocrinol Metab. 2013;98(8):E1410-E1413.

38. Agarwal AK, et al. AGPAT2 is mutated in congenital generalized lipodystrophy linked to chromosome 9q34. Nat Genet. 2002;31(1):21-23

39. Agarwal AK, et al. PSMB8 encoding the $\beta 5$ i proteasome subunit is mutated in joint contractures, muscle atrophy, microcytic anemia, and panniculitis-induced lipodystrophy syndrome. Am J Hum Genet. 2010;87(6):866-872.

40. Abecasis GR, Cherny SS, Cookson WO, Cardon LR. Merlin — rapid analysis of dense genetic maps using sparse gene flow trees. Nat Genet. 2002;30(1):97-101.

41. Davydov EV, Goode DL, Sirota M, Cooper GM, Sidow A, Batzoglou S. Identifying a high fraction of the human genome to be under selective constraint using GERP++. PLoS Comput Biol. 2010;6(12):e1001025. 